\title{
Ueber die angeborene Pigmentirung der Sclera und ihre pathogenetische Bedeutung.
}

\author{
Von \\ Prof. J. Hirschberg \\ in Berlin.
}

Hierzu Tafel I. II. III.

\begin{abstract}
Die menschliche Sclerotica ist in der Norm für die makroskopische Betrachtung pigmentfrei. Bei mikroskopischer Beobachtung findet man allerdings Pigmentzellen in derselben, jedoch nur spärlich, und zwar mit einiger Regelmässigkeit nur in der Nähe des Opticuseintritts und am Cornealfalz. ${ }^{*}$ )

Ausnahmsweise kommen aber recht ansehnliche angeborene Pigmentflecke in der menschlichen Sclera vor, welche dem befallenen Augapfel ein eigenthümlich geflecktes Aussehen verleihen. Der Zustand ist recht selten.
\end{abstract}

In dem grossen Sammelwerke von Graefe-Sämisch fehlt im Capitel von der Sclera**) jeder Hinweis, in dem

*) Vgl. Waldeyer, Graefe-Sämisch I, 216.

**) IV, 1 .

v. Graefe's Archiv für Ophthalmologie, XXIX, 1. 
der angeborenen Anomalien*) wird einfach nur citirt, dass v. Ammon angeborene Pigmentflecke in der Selera beobachtet habe. Eine gate Beschreibung finde ich in dem Atlas von R. Liebreich**), bei dem es folgendermassen beisst:

"Ich habe nur fünf Falle von Cyanosis bulbi gesehen; im Wesentlichen boten aile dasselbe Bild. Es zeigte sich dies bei allen fünfen nur an dem einen Auge, welches sich von dem andern durch die viel dunklere Farbe der Iris und die grauen in's Violette spielenden Flecke der Sclera unterschied (die in einiger Entfernung vom Rande der Hornhaut gruppenweise auftreten). Nur in einem von diesen fünf Fällen fand ich die Papille des Opticus selbst stark pigmentirt."

Nach meinen Beobachtungen kann ich diese Beschreibung nur loben, möchte aber den Namen der Cyanosis bulbi durch einen anderen, wie mir scheint, passenderen, den der fleckförmigen Melanosis sclerae***), ersetzt wissen.

Der Name Cyanosis bulbi sollte für jene seltenen Falle reservirt bleiben, von denen ich zwei beobachtet und noch drei in der ophthalmologischen Literatur $\dagger$ ) verzeichnet finde, wo bei angeborenem schwerem Herzfehler nicht blos die Cutis, sondern auch das Weisse im Auge, also die Conj. sclerae, vollkommen eyanotisch aussieht; und alle sichtbaren Venen im Augengrunde ganz enorm verbreitert erscheinen.

Die angeborene fleckige Melanose der Sclera habe ich selber nur wenige Male beobachtet und war

*) Vgl. Graefe-Sämisch II, 1, 118.

**) II. Aufl., 1870, p. 28.

****) Den auch Schmidt-Rimpler (Dissert. von Schaumburg, 1882) anwendet.

†) Leber in Graefe-Sämisch V, 524. 
einigermassen überrascht, als im letzten Sommer binnen vier Wochen drei Fälle, und zwar aus ganz verschiedenen Familien, sich mix vorstellten.

Der erste Fall war der einfachste. Ein Siebzehnjähriger kam wegen Lidrandentzündung. Patient ist brünett, aber seine rechte Iris ausserordentlich viel dunkler braun als die linke. Die rechte Sclera enthält rings um die Hornhaut grosse dunkelviolette Flecke, von denen er mit Bestimmtheit anzugeben vermag, dass sie angeboren sind. Der Augengrund ist beiderseits normal, aber rechts weit dunkler pigmentirt als links. Sehkraft beiderseits durchaus befriedigend.

Der zweite Fall ist zwar in seinem äusseren Habitus dem ersten vollkommen ähnlich, aber im Augeninnern durch eine merkwürdige, nur mit dem Augenspiegel sichtbare Geschwulstbildung complicirt.

Die 34jährige Patientin gelangte Mitte Juli 1882 zur Aufnahme wegen einer seit dem letzten Winter von ihr beobachteten Sehstörung auf dem linken Auge. Sonst fühlte sie sich vollkommen wohl. Ihre Haarfarbe ist dunkel. Das rechte Auge hat eine Iris von etwas unbestimmter, gemischter, graugrünlich-bräunlicher Färbung; normale Sehkraft und Augenspiegelbefund. Das linke Ange hingegen besitzt eine ganz dunkelbraune Iris und rings um die Hornhaut grössere, scharfbegrenzte, dunkelviolette Flecke in der Sclera, die theilweise fast zum Aequator reichen. (Vergl. Fig. 1.) Auf das Allerbestimmteste wird versichert, dass die Dunkelfärbung der Iris und die Fleckung des Weissen im linken Auge angeboren sei, bezw. seit der allerfrühesten Kindheit unverändert so bestehe.

Im Grunde dieses linken völlig reizlosen Auges fand ich eine Abweichung, die man am ungezwungensten als Neubildung am Sehnerveneintritt nebst Umgebung zu 
deuten im Stande ist. (Vgl. Fig. 2).*) Eine Geschwulst von verhältnissmässig beträchtlicher Grösse und Höhe erhebt sich in der Gegend des Sehnerveneintritts. Thre Erbebung über das Nivean der Netzhaut, mit dem Angenspiegel gemessen, beträgt mindestens $2 \frac{1}{2} \mathrm{~mm}$. Ihre Länge von rechts nach links ist auf $6 \mathrm{~mm}$, ihre Breite von oben nach unten auf $4^{1} / 2 \mathrm{~mm}$ zu schätzen. Die Masse der Neubildung ist wesentlich radiär gestreift; die Hauptblutgefässe sind streckenweise verdeckt, überwuchert; die Confluenzstelle derselben, der normale Sehnerveneintritt, nicht mehr sichtbar; diese Partie ist zart röthlich weiss, mit einigen neugebildeten Gefässreiserchen und hellen. Punkten versehen. Schläfenwärts sinkt hier die Prominenz ziemlich steil in das Niveau der Netzhaut hinab. Ein System zarter vertikaler regelmässiger Linien markirt uns daselbst eine fast mikroskopische Abhebung und Fältelung der Netzhaut**;), die hier auch von glitzernden Punkten durchsetzt erscheint.

Die Fovea centralis ist frei. Dem entspricht die fast normale centrale Sehschärfe des Auges. Dasselbe liest die feinste Schrift ganz bequem. Natürlich ist aber entsprechend der Geschwulstbildung ein grosses paracentrales Scotom rings um den normalen blinden (Mariotte'schen) Fleck entwickelt und setzt sich nach oben bis zur Grenze des Gesichtsfeldes fort. (Vergl. Fig. 3; das doppelt schraffirte bezeichnet den Defect im Gesichtsfelde.)

*) Die Zahlen sind Dioptrien der Correctionsgläser, mit denen ich die verschiedenen Theile der Geschwulst am dentlichsten sah. Mit $-4,5 \mathrm{D}$ sehe ich den emmetropischen Angengrund. Die Hinzufügung von je $+1 \mathrm{D} \mathrm{zu}-4,5$ bedentet immer eine Prominenz von etwa $0,3 \mathrm{~mm}$. macht.

**) Diese Beobachtung habe ich anch in anderen Fallen ge- 
Die Annahme, dass hier eine Geschwulst vorliegt und nicht ein Entzündungsproduct, wurde durch die weitere Beobachtung bestätigt, insofern binnen drei Monaten weder in dem Augenspiegelbefund noch in der Functionsstörung eine wesentliche Aenderung eingetreten ist.

Erst in der allerletzten Zeit begann die centrale Sehschärfe abzusinken, da die Neubildung der Fovea centralis, also der Gesichtsfelddefect dem Fixirpunkt, in bedenkTicher Weise nahe rückte. Am 8. November 1882 erKannte das betreffende Auge nur noch Sn CC:15' und las mit +6 Zoll Sn $3 \frac{1}{2}$ mühsam; das Scotom reichte im horizontalen Meridian vom 4. bis zum 40. Grad lateralwärts vom Fixirpunkt. 18. December 1882: Finger in $10^{\prime},+6 " \mathrm{Sn} 3^{1 / 2}$ sehr mühsam. Gesichtsfeld wie zuvor. Stärkste Prominenz $=3 \mathrm{~mm}$. Transitorische Amaurose des linken Auges, täglich 10-12 Mal, von je 1 Minute Daner.

Auflösende und ableitende Mittel waren ohne merklichen Einfluss.

Die histologische Natur der Neubildung kann aus dem Augenspiegelbefund nicht erschlossen werden, wenn nicht vollständige, d. h. bis zur mikroskopischen Untersuchung durchgeführte Parallelerfahrungen in genügender Anzahl die Diagnose stützen. Deshalb hielt ich mich vorläufig nicht für berechtigt, der Patientin die Enucleation des noch functionirenden Auges anzurathen, obwohl ja späterhin eine Periode kommen kann, wo dieser Rath geboten erseheint.

Der dritte Fall ist dem zweiten insofern ähnlich, als auch er mit einer intraocularen Geschwulst complicirt orscheint, und jedenfalls der interessanteste von allen, weil or zur anatomischen Untersuchung des befallenen Augapfels gelangte. 
Eine 56jährige Frau kam am 4. August 1882 wegen einer seit längerer Zeit (Pfingsten d. J.) beobachteten schmerzlosen Erblindung ihres rechten Auges.

Das Haar der Patientin ist dunkel, obwohl schon stark ergraut. Die Iris des rechten vollkommen gesunder. und sehkräftigen Auges ist grünlich grau, die des linken vollkommen erblindeten ist ganz dunkelbraun.

Die Bindehaut des linken Augapfels zeigt nahe dem Hornhautrande vier dunkelbraune, $3-4 \mathrm{~mm}$ breite Pigmentflecke. Ausserdem schimmern einige scharf umschriebene, weit grössere, dunkelviolette Flecke der Selera durch, welche hauptsächlich in der Aequatorialgegend sitzen. jedoch zum Theil bis nahe an den Hornhautrand herankommen.

Patientin versichert auf das Allerbestimmteste, dass diese Flecke angeboren seien; nur sollen sie in der allerersten Lebenszeit mehr röthlich ausgesehen haben. *)

Der Augenspiegel zeigt feine beginnende Linsentrübung und im Centrum des Augengrundes eine stark vorragende, zum Theil mit Blat-Gefässen und Flecken bedeckte, röthliche Nenbildung, von deren Kuppe aus eine zeltartige Netzhaut-Ablösung nach allen Richtungen hin der Ora serrata zustrebt. Spannung normal, der Augapfel frei von Reizung.

Ich entwickelte den anwesenden Aerzten (u. A. Herm Herrn Collegen Berger aus Graz) die Diagnose eines aus. angeborener Pigmentirung hervorgegangenen Aderhautsareoms und rieth der Patientin die Enucleation an.

Erst am Vormittag des 21. October 1882 kehrt sie wieder und bittet um die sofortige Entfernung des Aug-

*) Beide Patientinnen (Fall 2 und 3) erzählen eine Historie vom "Versehen" ihrer Mutter. 
apfels, da sie seit zwei Tagen von furchtbaren, geradezu unerträglichen Sehmerzen in dem erkrankten Auge gepeinigt sei und auch das gute nicht mehr gehörig offnen könne.

Das letztere thränt, hat aber noch normale Sehkraft und Spiegelbefund. Das linke zeigt Lidschwellung, Pericornealinjection sowie die Spuren einer schmalen unregelmässigen Iridectomie nach oben. (Am 25. August war sie nämlich inzwischen anderwärts operirt worden.) Die Gegend der peripheren Wunde ist prominent, die Linse vollständig getrubt, der Bulbus steinhart und dabei auf Druck sehr empfindlich. Ich schritt sofort zur Enucleation und nahm dabei die Augapfelhindehaut bis zum Aequator fort. Nach 8 Tagen wurde die Patientin in durchans befriedigendem Zustand entlassen. (Januar 1883: Status idem.)

Die anatomische Untersuchung des enucleirten Augapfels zeigt (vgl. Fig. 4), dass die aus Strichen zusammenfliessenden melanotischen Flecke der Sclera nach hinten uber den Aequator fort bis zum Sehnerven reichen, der selber von Melanose frei, aber im grössten Theil seines Querschnitts grau atrophiseh erscheint. Auch an der Austrittsstelle der Wirbelvenen (vgl. Fig. 4) ist die Sclerotica stärker pigmentirt.

Der Augapfel wird im horizontalen Meridian durchsehnitten. Länge wie Breite (einschliesslich der selerocornealen Kapsel) misst etwa $25 \mathrm{~mm}$. Die Hauptreränderung (vgl. Fig. 5, welche die Schnittfläche der oberen Hälfte in etwa zweifacher Vergrösserung darstellt), besteht in einer polypös geformten, partiell pigmentirten Geschwulst, welche mit breiter Basis in der Gegend des hinteren Augenpoles aus der Aderhaut entspringt, den Sehnerveneintritt überlagert, die zu einem Faltenpaar zusammengepresste Netzhaut emporhebt und bis über den 
Mittelpunkt des Auges nach vorne dringt. Die Grundfläche der Geschwulst, welche mit der Aderhaut verwachsen ist, misst etwa $9 \mathrm{~mm}$, die grösste Breite fast $12 \mathrm{~mm}$, die grösste Länge $14 \mathrm{~mm}$, also nahezu $2 / 3$ der inneren Augenachse. Die Schnittfläche der Neubildung hat eine ganz leicht gelappte Contourlinie, und eine theils graugelbe, theils dunkle und selbst tintenschwarze Färbung, über deren Vertheilung ein Blick auf die Abbildung der Schnittfläche besser belehrt als eine längere Beschreibung.

Ueber den Ursprung der Neubildung aus der Aderhant kann kein Zweifel obwalten. Die ganze Netzhaut ist erhalten, nur in zierliche Falten durch die Steigerung des intraocularen Druckes zusammengepresst; sie haftet überhaupt nur durch einen dünnen Stiel am Sehnerveneintritt und in einer schmalen Linie an der Ora serrata; aber auch der Ciliargegend liegen 2 Platten der Netzhaut an, von denen die vordere die Hinterfläche der Linse überzieht. Dort findet sich ein kleiner viereckig begrenzter Raum zwischen Linse und Geschwulstkuppe, ganz von Netzhaut austapeziert, der Rest des Glaskörperraumes.

Eigenthümlich ist noch, dass fast die ganze Papilla optica von der medialen Contourlinie der Neubildung dicht uberlagert wird, was ich zur Zeit noch in einem klinischen Fall zu beobachten Gelegenheit habe. Der ganze Uvealtractus, Iris, Ciliarkörper und Aderhaut, ist ungewöhnlich dunkel, sonst aber vollständig, bis auf die Basalstelle des Tumor. Die Linse ist getrübt und geschrumpft, wohl bei der Iridectomie verletzt.

Die Sareomstruetur der Neubildung war schon nach dem makroskopischen Verhalten nicht za bezweifeln und in den Mikrotomschnitt-Präparaten, die mein damaliger Assistent, Horr Dr. F. Krause, angefertigt hat, leicht zu erkennen. 
Eine $0,3 \mathrm{~mm}$ breite tintenschwarze Lage, welche nach beiden Seiten direet in die dunkle Aderhaut ubergeht und an den Vebergangsstellen Blutgefässlücken zeigt, bildet die Basis der Geschwulst und schlägt sich beiderseits auf die Seitenflächen, wenigstens auf eine Strecke von $4-5 \mathrm{~mm}$, hinüber.

Nach innen - gegen das Gesehwulstgewebe - lockert sich diese dichte melanotische Schicht und sendet einige vertical emporstrebende Züge aus (zwei parenchymatöse, zwei seitliche, einen an jeder der beiden Seitenflächen); so werden in den tiefsten Theilen der Neubildung Netze von Pigmentzellen erzeugt, in deren Maschenräumen die kleineren ungefärbten Zellen liegen.

In den mittleren Theilen der Neubildung treten die dunklen Pigmentzellen, die schön entwickelte Spindel- und Stern-Formen und beträchtliche Grösse $(40-60 \mu)$ zeigen, mehr zurück; sie bilden innerhalb der dichtgedrängten pigmentlosen Zellen nicht mehr Netze, sondern nur unvollständige vertical stehende Septa; sie liegen auch in der dünnen Wandung der zahlreichen Netzbautgefässe, einzelne sogar, wie es scheint, in deren Lumen. Hier und da sind auch Gruppen kleinerer $(10-20 \mu)$ mehr rundlicher und hellbrauner Pigmentzellen anzutreffen.

In den pigmentlosen Theilen der Neubildung (vergl. Fig. 7, Vergrösserung 275:1) sieht man zahlreiche, schmale and breite, wandungslose oder ganz dünnwandige, noch gefüllte Blutgefässe, um welche sich die dichtgedrängten Zellen fast ohne Zwischengewebe bündelweise gruppiren. Die vom Zellleib eng umschlossenen Kerne sind leicht oval oder elliptisch, etwa $6-8 \mu$ lang.

Auf einem Uebersichtsschnitt-Präparat dringt ein rundlicher, durchweg aber mässig (durch kleinere Zellen) pigmentirter Knoten an die freie Obertläche der medialen Seite. Hierselbst ist die Netzhaut durch schräg parallelfaseriges Gewebe mit der Nenbildung verwachsen. Die 
Retina ist frei von Geschwulstzellen, kernreich, an der Innenfläche faserig. Die letztere liegt dicht an der zweiten Platte der abgelösten Netzhaut, die etwas dünner ist und gleichfalls aus einer inneren faserigen und äusseren körnigen (zellenreichen) Lage besteht.

An der lateralen Seite des Tumor sieht man in einiger Entfernung von der Basis unter- wie oberhalb der pigmentirten Grenzschicht ein mächtiges Blutcoagulum. Kleinere Blutungen trifft man auch inmitten des Geschwulstgewebes. Die Aderhaut (nebst Ciliarkörper und Iris) ist so dunkel pigmentirt, dass man auf den Dickendurchschnitten ihre Structur nicht zu erkennen vermag. Die Linse enthält Faserreste und reichliche Myelintropfen.

In der Sclerotica sieht man auf dem Durchschnitt kürzere und längere schwarze Linien, theils oberflächlich, theils tiefer dem weissen Fasergewebe eingelagert. Die längste ist in der Gegend der Geschwulstbasis, aber von der letzteren noch immer durch weisse Scleroticalmasse geschieden. Mikroskopisch bestehen diese dunklen Linien der Sclera lediglich aus normalen gestreckten Pigmentzellen. (Vergl. Fig. 8, Vergrösserung 100:1 und Fig. 6, erstere stellt einen Flächen-, letztere einen Dicken-Schnitt dar). Hier und da ist die Pigmentanhäufung aber so dicht, dass man sie nicht $\mathrm{zu}$ differenziren vermag.

In der ganzen mir zugänglichen Literatur konnte ich nur einen wirklich analogen Fall von Hulke in London aus dem Jahre 1860 auffinden, den auch Fuchs citirt, welcher 259 Fäle von Aderhautsareom zusammengestellt hat.

Hulke*) beobachtete eine 62jährige Frau, welche eine angeborene dunkle Pigmentirung der Haut der

*) Ophth. Hosp. Rep. III. 179 a 1860. In der Figur-Erklärung sind einige Druckfehler. 
rechten Augenbrane und der Lider zeigte, sowie schwarze Flecken auf der Sclera und seit $1 \frac{1 / 2}{2}$ Jahren über Sehstörung zu klagen hatte. Eine dunkle Geschwulst war im Augengrunde nachweisbar. Schmerzen stellten sich ein. Die Iridectomie war erfolglos, die Enucleation wurde verrichtet. Die Geschwulst entsprang aus den mittleren Aderhautschichten, aussen-unten-hinten im Augengrunde, und reichte bis zum Centrum des Augapfels nach vorn. Der Sehnerv war an der Durchschnittsstelle pigmentirt, die Netzhaut total abgelöst, die schwarzen Flecke in der Sclera waren einfache Pigmentirungen.

Dass angeborene Pigmentflecke der Cutis im späteren Alter den Ausgangspunkt ron melanotischen Geschwülsten abgeben, ist genügend bekannt and auch von Virchow in seiner Onkologie (II. 122, 234,272 ) ausführlich erörtert.

Hier kann man in manchen Fällen präventiv verfahren, nämlich die pigmentirten Flecke der Haut bereits in der Jugendzeit der Patienten entfernen. Weniger leicht geht dies schon an bei den angeborenen umschriebenen schwarzen Flecken der Iris, aus denen im höheren Alter mitunter Irissarcome entstehen: von den 16 Fällen von Irissareom, welche in der Literatur vorgefunden werden, waren drei aus angeborenen Pigmentflecken der Regenbogenhaut hervorgegangen, darunter einer, den ich selber beschrieben.

Vollends unausführbar ist die präventive Therapie bei diesen angeborenen Pigmentflecken der Sclera; dieselben stellen überhaupt nur eine Begleiterscheinung dar, ein Symptom der eigentlichen Abnormität, nämlich jener starken diffusen Pigmentirung des ganzen Uvealtractus, die klinisch in der dunklen Färbung der Iris und, wenn man den Augenspiegel anwendet, auch des Augengrundes sich kundthut. 
Bei der räthselhaften Aetiologie der Aderhautsarcome (und der sarcomatösen and krebsigen Geschwülste überhaupt) sind derartige Falle, wenn sie zunächst auch nur eine kleine Minorität ausmachen, doch recht belehrend, weil sio zeigen, dass die Disposition zu einer im mittleren oder selbst im Greisen-Alter auftretenden progressiven Nenbildung schon während des Foetallebens angelegt sein kann. 


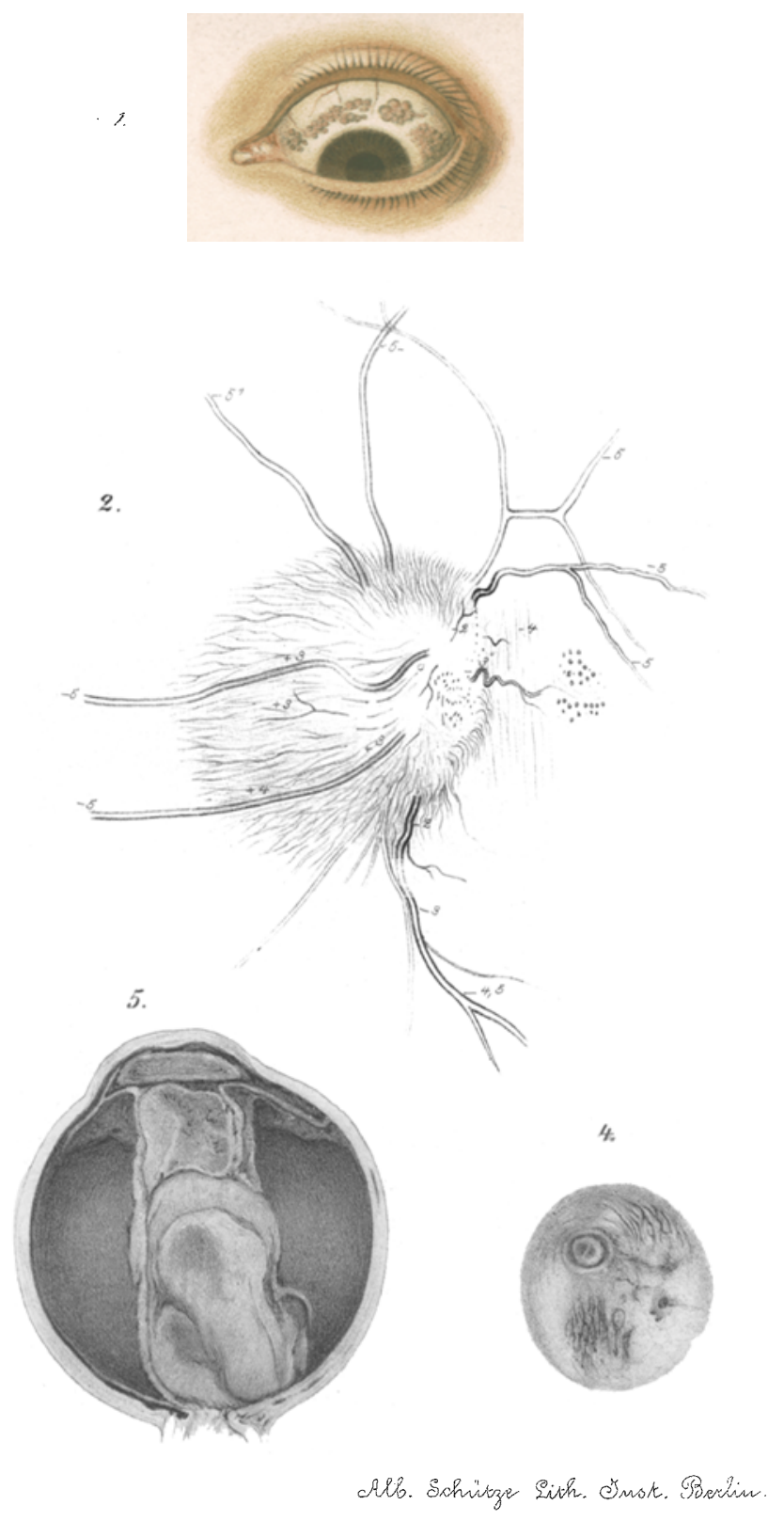




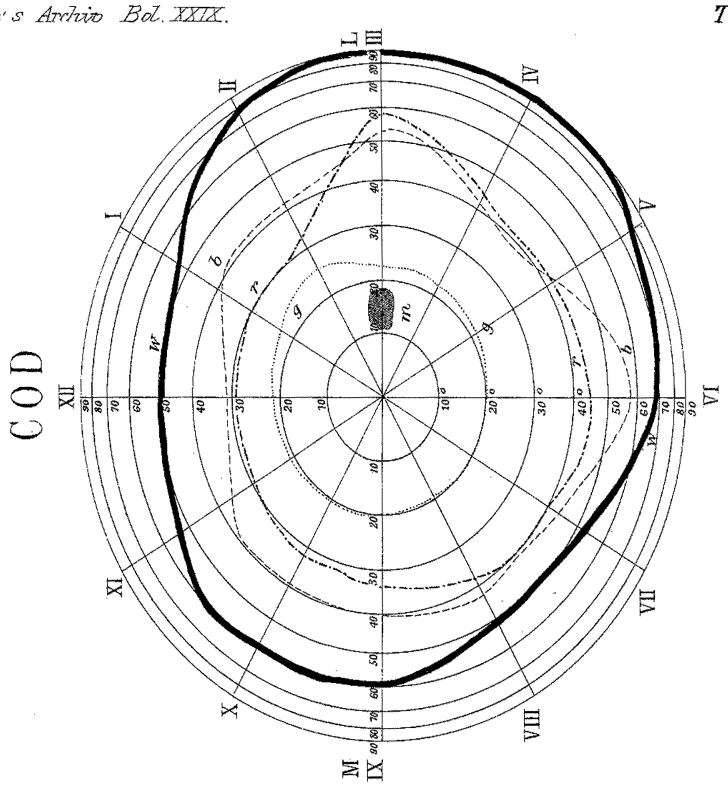

Tag. II.

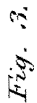

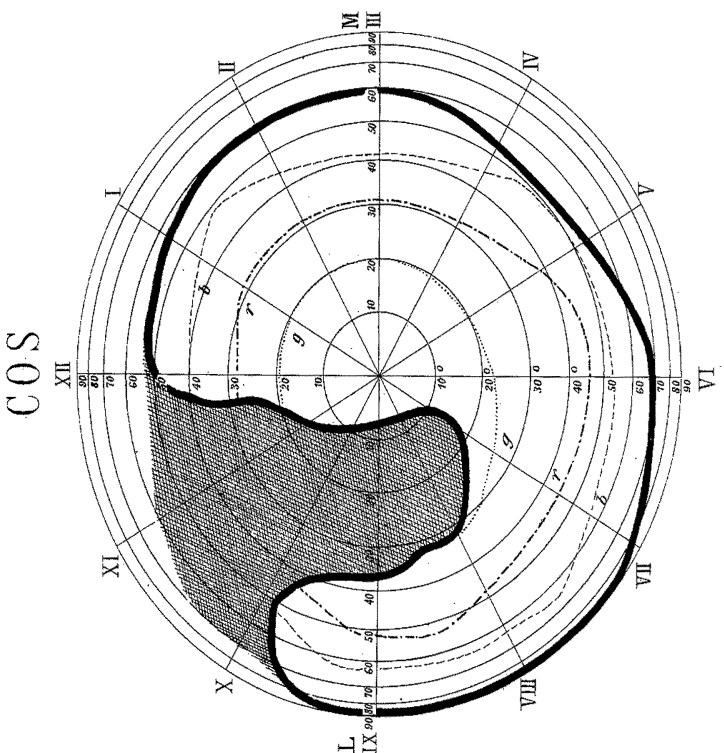

cllbert schütze Sith. Cinst. 


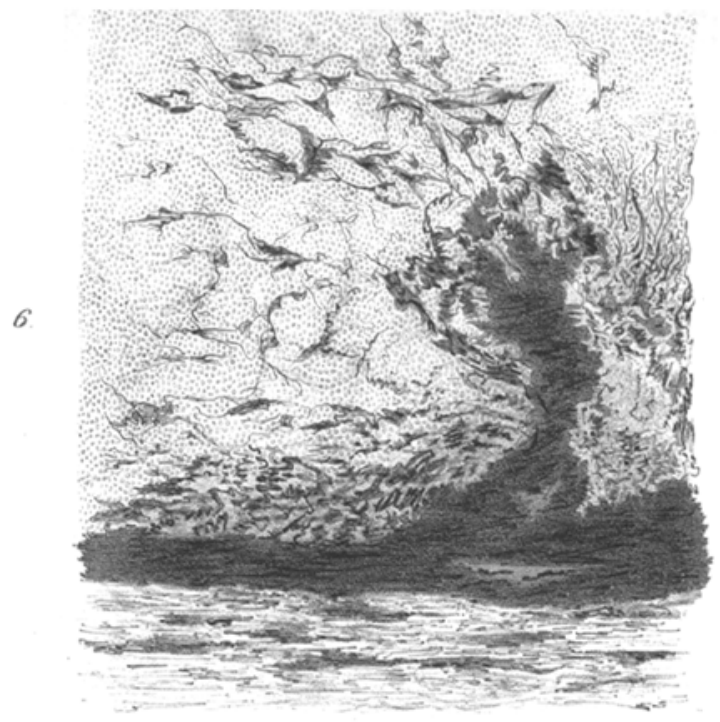

8 .

7.

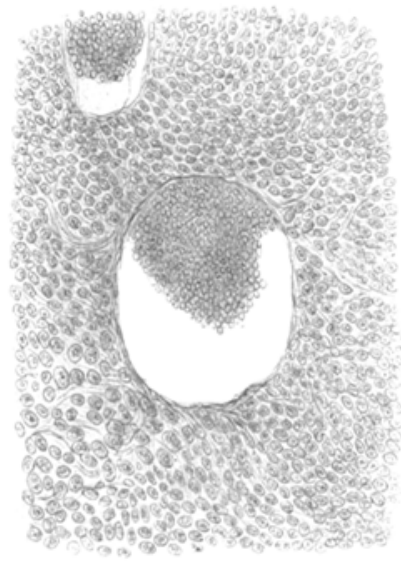

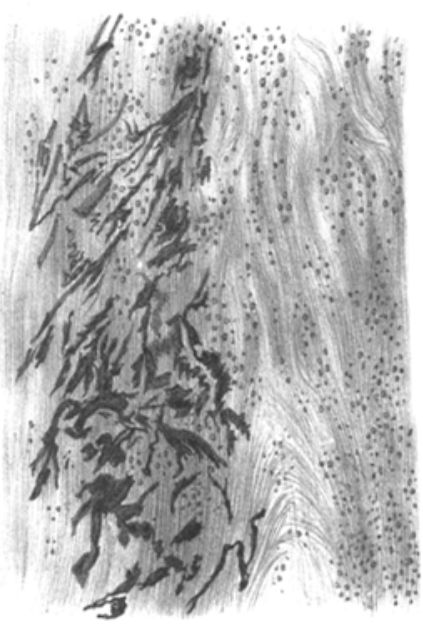

